\title{
Antagonizing the effects of Bst-2/tetherin: multiple ways to accomplish a common goal Klaus Strebel
}

\author{
Address: Viral Biochemistry Section, LMM, NIAID, NIH; Bldg. 4/310, 4 Center Drive MSC 0460, Bethesda, MD 20892-0460, USA \\ from Frontiers of Retrovirology: Complex retroviruses, retroelements and their hosts \\ Montpellier, France. 21-23 September 2009 \\ Published: 24 September 2009 \\ Retrovirology 2009, 6(Suppl 2):II0 doi:10.1 I86/I742-4690-6-S2-II0
}

This abstract is available from: http://www.retrovirology.com/content/6/S2/II0

(c) 2009 Strebel; licensee BioMed Central Ltd.

The HIV-1 Vpu protein has long been known to enhance the release of virions from infected cells and to induce proteasomal degradation of the $\mathrm{CD} 4$ receptor. While the mechanism of CD4 degradation has been reasonably well explored, our mechanistic understanding of how Vpu facilitates virus release has remained vague. A breakthrough came with the recent identification of Bst-2 (also referred to as CD317 or tetherin). Bst-2 is a host factor whose expression is associated with the inhibition of HIV1 virus release. Interestingly, $\mathrm{Vpu}$ is not the only viral accessory protein with the ability to overcome the inhibitory effect of Bst-2. Indeed, some HIV-2 isolates, although lacking a $v p u$ gene, have been known to encode a Vpu-like activity in their Env glycoprotein and were now shown to antagonize Bst-2. Very recently, several SIV Nef isolates have also been associated with enhanced virus release through inhibition of Bst-2 function. Thus, there appear to be at least three retroviral proteins targeting Bst-2. Interestingly, these are the same three viral proteins that can target CD4. We have started a functional analysis to understand at what level HIV-1 Vpu and HIV-2 Env proteins interfere with Bst-2 function to determine if the mechanisms for targeting CD4 and Bst-2 are the same or not. Consistent with published reports we find that Vpu can induce surface down modulation of Bst-2 and cause degradation of Bst-2. However, Vpu can also facilitate virus release in the absence of detectable Bst-2 surface down modulation and degradation and in that respect resembles the HIV-2 Env protein. Possible mechanisms of Bst-2 inhibition by HIV-1 Vpu and HIV-2 Env will be discussed. 